\title{
Cimitero di Atella : résultats de la mission 2019
}

Cimitero di Atella: results of the 2019 fieldwork

Roxane Rocca, Amélie Da Costa, Lucie Germond et Daniele Aureli

\section{(2) OpenEdition}

Journals

\section{Édition électronique}

URL : http://journals.openedition.org/cefr/4436

DOI : $10.4000 /$ cefr.4436

ISSN : 2282-5703

Éditeur

École française de Rome

Référence électronique

Roxane Rocca, Amélie Da Costa, Lucie Germond et Daniele Aureli, « Cimitero di Atella : résultats

de la mission 2019 ", Chronique des activités archéologiques de l'École française de Rome [En ligne], Italie du Sud, mis en ligne le 14 juillet 2020, consulté le 14 juillet 2020. URL : http://journals.openedition.org/ cefr/4436; DOI : https://doi.org/10.4000/cefr.4436

Ce document a été généré automatiquement le 14 juillet 2020

(c) École française de Rome 


\section{Cimitero di Atella : résultats de la mission 2019}

Cimitero di Atella: results of the 2019 fieldwork

Roxane Rocca, Amélie Da Costa, Lucie Germond et Daniele Aureli

\section{NOTE DE L'AUTEUR}

Les nouvelles recherches menées à Atella sont financées par l'École française de Rome, dans le cadre de l'axe 2 du projet quinquennal PALEO. Les recherches sur place sont soutenues par la Commune d'Atella et la région Basilicate. L'autorisation de fouille et d'étude du matériel nous est confiée par la Soprintendenza Archeologia Belle Arti e Paesaggio della Basilicata, concession MIBACT, DGABAP n ${ }^{\circ} 17811 \mathrm{du}$ 27/06/2019. Les cahiers de fouilles, ainsi que les anciennes collections, sont conservés au Museo archeologico nazionale del Melfese, et accessibles grâce à la bienveillance du Professeur E. Borzatti von Löwenstern. Les analyses et missions de terrains sont prises en charge par les institutions des chercheurs qui collaborent au projet :

Jean-Jacques Bahain (UMR 7194 HNHP) ; Paolo Beneduce (Università degli Studi della Basilicata) ; Francesco Boschin (Università degli Studi di Siena) ; Paola Di Leo (CNR IMAA) ; Federico Di Rita (Sapienza Università di Roma) ; François Fouriaux (École française de Rome) ; Paolo Giannandrea (Università degli Studi della Basilicata) ; Salvatore Ivo Giano (Università degli Studi della Basilicata) ; Dario Gioia (CNR - IBAM) ; Donatella Magri (Sapineza Università di Roma); Sebastien Nomade (UMR 8212, LSCE); Alison Pereira (École française de Rome) ; Raffaele Sardella (Sapienza Università di Roma) ; Marcello Schiattarella (Università degli Studi della Basilicata); Roberto Sulpizio (Università degli Studi di Bari Aldo Moro) ; Pierre Voinchet (UMR 7194 HNHP); Maurizio Zambaldi (Università degli studi di Trento). Nous remercions également chaleureusement l'équipe de fouille ainsi que les habitants d'Atella. 


\section{Introduction}

1 Les travaux en cours sur le site paléolithique de Cimitero di Atella s'inscrivent dans un projet de recherche plus large qui vise à reconstituer les dynamiques de peuplement au Paléolithique ancien en Italie. En effet, les travaux précédents, menés pendant une vingtaine d'années par E. Borzatti ${ }^{1}$, avaient conduit à attribuer ce site à l'Acheuléen archaïque, période encore peu connue qui marque le début de l'émergence d'un nouveau type d'outils en Europe, le biface. Dans un souci de conservation, mais aussi pour pallier le manque de connaissance sur cette période charnière du peuplement préhistorique de l'Europe, un nouveau projet de recherche franco-italien a été lancé. Les premières années ont été consacrées à la révision du contexte chronostratigraphique $^{2}$, au réexamen du matériel archéologique ${ }^{3}$ et à la fouille partielle de plusieurs niveaux ${ }^{4}$. La mission 2019 avait pour objectif d'évaluer l'extension du site et de permettre la mise en place d'une fouille extensive à travers l'ouverture d'une nouvelle aire. Cet objectif a été atteint et nous permet désormais d'avoir une vision globale du potentiel archéologique de ce gisement.

\section{Présentation du site}

\section{Problématique archéologique}

2 Le site de Cimitero di Atella par sa position géographique et son attribution chronologique, aux alentours de 600000 ans, se situe à un moment clé du Paléolithique ancien européen ${ }^{5}$. En effet, cette période représente un moment charnière entre la première vague des peuplements de l'Europe qui remontent à plus de 1 million d'années ${ }^{6}$ et l'émergence du phénomène technique bifacial entre 700000 et 500 $000 \mathrm{ans}^{7}$. Cette phase, aussi appelée Early Acheulean, est principalement documentée dans le sud de l'Europe et demeure mal connue (fig. 1). Si les données sur le Paléolithique de la Basilicate sont ténues, cette région est paradoxalement centrale à cette période particulière, puisqu'un autre site, Notarchirico à Venosa, est également rapporté à cette phase ancienne de l'Acheuléen ${ }^{8}$. La présence à la fois à Atella et à Notarchirico de niveaux d'origine volcanique, liés à l'activité éruptive du mont Vulture, permet de dater ces occupations avec une précision chronologique rarement atteinte pour ces périodes 9 . De plus, la pluralité des chaînes opératoires représentées (bifaces, mais aussi petits outils et débitage) nous offre l'opportunité d'aborder non seulement la question de l'émergence du phénomène bifacial mais aussi de replacer cet outil dans son système technique ${ }^{10}$. En effet, la prégnance du débat autour des mécanismes d'apparition du biface en Europe (diffusion ou convergence?) a eu tendance à concentrer l'attention uniquement sur cet outil et à l'extraire de son contexte technique. La présence d'un niveau archéologique d'occupation relativement bien conservé à Atella ${ }^{11}$, ainsi que la mise en place d'une méthode d'analyse permettant une étude de toutes les composantes du système technique permettra donc d'apporter de nouvelles informations sur les changements techno-culturels à cette période clé du peuplement de la Méditerranée. 
Fig. 1 - Principaux sites en Italie entre 700000 et 500000 ans.

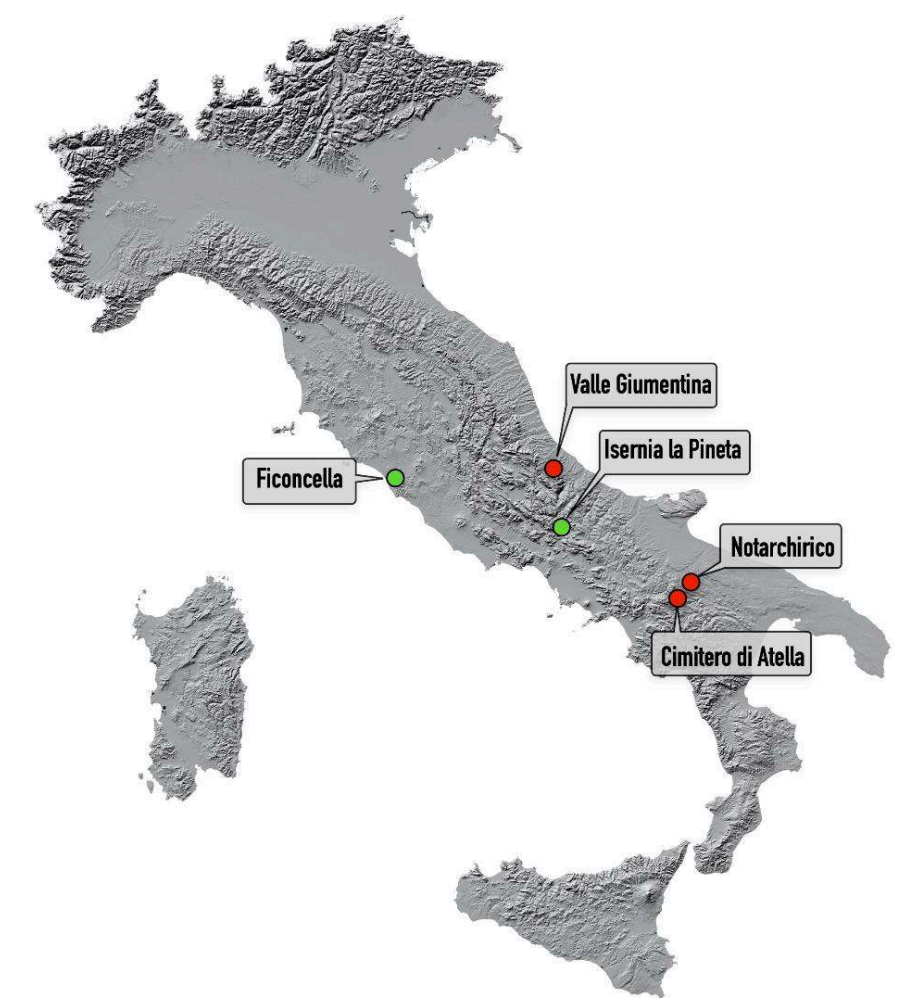

En rouge, sites avec bifaces, en vert sans bifaces.

R. Rocca.

\section{Fouille Borzatti}

Après sa découverte dans les années 1990, la fouille a été dirigée par le Professeur E. Borzatti pendant plus de 20 ans. Les nombreuses publications sur le site et la région ont permis d'établir un cadre chrono-stratigraphique de référence pour la région et de rapporter le site d'Atella au Paléolithique inférieur ${ }^{12}$. Cette attribution à environ 600 000 ans s'avérait également cohérente avec les restes de faunes attribués à Palaeoloxodon antiquus ainsi qu'à Bos primigenius et à différentes espèces de cerfs ${ }^{13}$. La présence de "bifaces » au sein de l'outillage lithique a conduit les auteurs à attribuer Cimitero di Atella à l'Acheuléen archaïque ${ }^{14}$. Au milieu des années 1990, une structure métallique qui se voulait temporaire a été édifiée afin de protéger le site tout en permettant qu'il soit visible du grand public (fig. 2).

Fig. 2 - Vue générale du site, de l'ancienne structure et de la nouvelle aire.

Image 10000000000003EB000002F146B656D95EF2E698.jpg

Photo R. Rocca EFR/Sabap Basilicata.

\section{La reprise des travaux à Atella}

4 Après cette première phase des recherches à Atella, le site a été abandonné pendant quelques années. La reprise des travaux en 2014 a donc été motivée non seulement par 
des aspects scientifiques, mais aussi par des questions de conservation, le matériel notamment faunique étant encore en place sur la fouille ${ }^{15}$.

Dans un premier temps, il s'est avéré nécessaire de confirmer l'attribution chronostratigraphique du site et l'interprétation culturelle du matériel archéologique (fig. 3). Concernant le cadre chronostratigraphique, les premières observations permettent de confirmer l'attribution préliminaire qui avait été proposée par E. Borzatti à environ 600000 ans, sur base des résultats des datations absolues ${ }^{16}$ et des corrélations avec la séquence type du Vulture ${ }^{17}$. D'un point de vue stratigraphique, cinq grands épisodes ont été enregistrés dans le bassin d'Atella au cours du Pléistocène moyen ${ }^{18}$ :

- Présence d'un lac contenant des sédiments volcaniques (niveaux inférieurs).

- Assèchement progressif du lac et mise en place d'un environnement plus marécageux. C'est au cours de cet épisode qu'a lieu la première fréquentation du site par les groupes humains (base ensemble $\mathrm{F}$ ).

- Un ou plusieurs épisodes de colluvions probablement liés à l'activité éruptive du Vulture (ensemble F).

- Un niveau de cendre volcanique (tephra I).

- Mise en place d'un cours d'eau, dont témoigne l'accumulation de dépôts de sables fins à moyens, qui alternent avec des dépôts d'origine éruptifs (ensemble L).

Fig. 3 - Log stratigraphique synthétique.

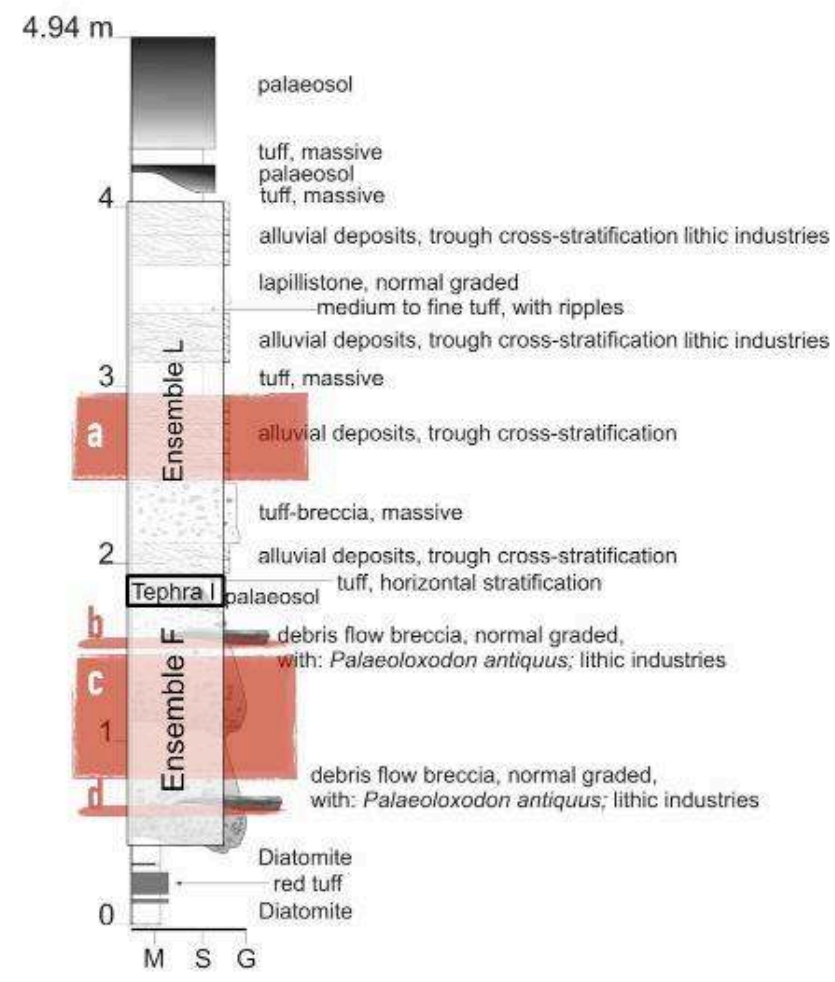

En rouge, principaux niveaux : a. niveau fluviatile inférieur (All 2) ; b. niveau base tephra I (Deb1) ; c. niveaux de colluvion (Deb 2) ; d. niveau de base du colluvion Deb 3).

Modifié d'après Gianandrea dans Rocca et al. 2016.

6 Concernant l'industrie lithique, la reprise de l'étude technologique du matériel issu des fouilles Borzatti, ainsi que les premières observations sur le matériel des fouilles 2016 et $2017^{19}$ ont permis d'apporter de nouveaux éléments sur la collection issue des 
anciennes fouilles et de nuancer certaines interprétations. Trois chaînes opératoires principales ont été identifiées. Les indices d'une chaine opératoire de confection de

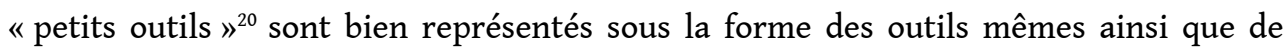
petits éclats d'encoches et de retouches caractéristiques. Une chaîne opératoire de débitage a également été identifiée. Le nombre des «macro-outils» découverts en contexte stratigraphique dans le niveau $\mathrm{F}$, a été considérablement réduit. Ainsi, outre la présence de rares bifaces, le débitage d'éclats et la confection de petits outils semblent représenter une part importante des assemblages de nombreux sites entre 800 et 400 000 ans dans le sud de l'Europe ${ }^{21}$.

7 En ce qui concerne l'assemblage faunique, les observations préliminaires sur les collections anciennes et les dernières années de fouille indiquent que le matériel est très fragmenté et contient donc peu de restes déterminables. La présence de Palaeoloxodon, de cervidés, de daims et de bovidés est confirmée, même si la distinction entre Bos et Bison est encore à démontrer.

8 Afin d'étendre la fouille, d'offrir de meilleures conditions de conservation et valoriser le site, un projet de «micro-musée » a été conçu en collaboration avec des institutions locales, nationales et européennes (Soprintendenza Archeologia Belle Arti e Paesaggio della Basilicata, commune d'Atella, région Basilicate). La première étape de ce projet, à savoir l'ouverture d'une nouvelle aire de fouille, a été atteinte en 2019.

\section{Résultats de la campagne 2019}

\section{Une nouvelle aire}

9 Un des objectifs de la mission de cette année était d'évaluer l'extension des dépôts. Un carottage réalisé en 2015 avait permis de mettre en évidence la présence d'une séquence similaire à celle présente dans l'aire Borzatti. Il a été donc été décidé d'ouvrir une nouvelle zone à l'est de l'ancienne structure (fig. 2). Cette nouvelle aire a été divisée en deux zones nord et sud, séparées par un témoin d'un mètre de large (fig. 4). Trois sondages principaux ont été réalisés, un dans la zone nord et deux dans la zone sud dans le but d'avoir un aperçu global de la séquence. 
Fig. 4 - Plan de l'aire Borzatti et de la nouvelle aire de fouille 2019.

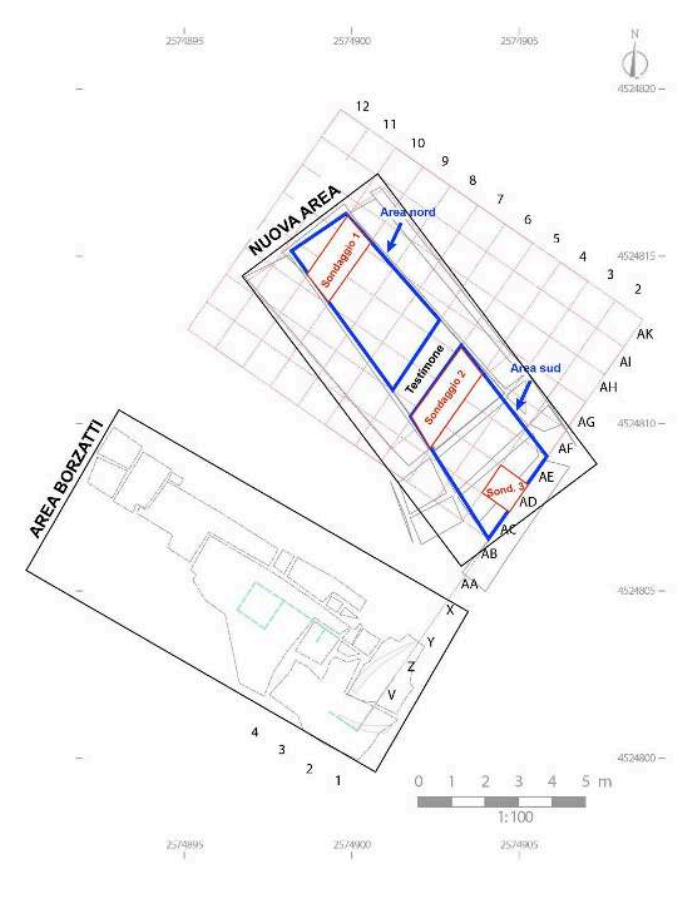

Modifié d'après Fr. Fouriaux/EFR Sabap Basilicata.

10 L'observation préliminaire de la stratigraphie des trois sondages principaux a permis de proposer une corrélation entre la séquence de la nouvelle aire et celle de l'aire Borzatti. Dans le sondage 1, un premier niveau de sédiments d'origine fluviatile, qui semble absent dans l'aire Borzatti, a été identifié (All 0). Deux autres niveaux de sables (All 1 et All 2), entrecoupés de deux niveaux de tephra (Vul 1 et Vul 3) qui semblent correspondre aux niveaux présents dans la séquence de l'aire Borzatti. Dans le sondage 2 , sous les niveaux historiques, un premier niveau de tephra ( $\mathrm{Vul} \mathrm{3}$ ) reposait sur un niveau de sables fluviatiles (All 3) correspondant probablement au L3 de l'aire Borzatti. Dans le sondage 3, nous avons intercepté deux niveaux d'origine fluviatile (All 3 et All 4), entrecoupés d'un niveau d'origine volcanique (Vul 4) et à la base du sondage, le tephra I.

En synthèse, il a été possible de repérer la plupart des niveaux présents dans l'aire Borzatti dans la nouvelle aire. Les niveaux supérieurs d'âge historique semblent témoigner d'une occupation lucanienne d'après les tessons céramiques, hypothèse qui devra être confirmée par des experts de la période. Concernant la séquence pléistocène, la partie supérieure présente dans les sondages 1 et 2 correspondrait à l'ensemble $\mathrm{L}$ de l'aire Borzatti, qui comporte une alternance entre des sables et des sédiments volcaniques probablement d'origine fluviatile. Ces niveaux sont pauvres en matériel archéologique, bien que quelques très petits éléments soient présents, notamment de petits éclats de retouche et de confection. Nous aurions donc affaire soit à des occupations très courtes et répétées dans le temps, soit au résultat d'un phénomène post-dépositionnel conduisant à un tri des éléments les plus légers. La partie basse de la séquence, identifiée dans le sondage 3, correspondrait à l'ensemble $\mathrm{F}$ de Borzatti qui comprend les niveaux sous-jacents au tephra I. Ces différents niveaux sont 
relativement riches en matériel archéologique (faune et lithique) et présentent une bonne qualité de conservation. Ce sont donc ces niveaux qui feront par la suite l'objet principal des recherches à Atella comme nous l'avions déjà présumé en 2018.

\section{Test tracéologique}

12 L'objectif de cette première étude tracéologique du matériel lithique du site d'Atella consistait en priorité à évaluer le potentiel de conservation des traces et plus précisément de noter s'il existait des états de conservation différents selon les niveaux archéologiques ou en fonction des matières premières des outils. Cette observation préliminaire du matériel lithique a été réalisée au moyen d'un microscope numérique Dino-lite (High Speed real time), avec une capacité de grossissement de 10x à 220x et muni d'un filtre polariseur permettant de moduler l'incidence de la lumière et d'améliorer la visibilité des matériaux riches en cristaux de quartz très réflecteur.

13 L'utilisation de ce microscope numérique a été privilégiée pour sa mobilité sur les différents lieux de mission. Il permet essentiellement d'observer les différents états de surface sur les pièces et d'orienter les premières recherches, notamment sur l'état des bords tranchants, la présence d'esquillements, d'émoussés ou d'écrasements pouvant relever d'une utilisation. En revanche, les polis et stries sont difficilement décelables par ce biais, sauf s'ils sont d'une ampleur notable.

14 Un premier échantillonnage, assez large, du mobilier a été réalisé en se basant sur les caractères techno-fonctionnels ${ }^{22}$ des pièces provenant des niveaux F-Deb 1 et F-Deb 2 de l'ancienne aire de fouille et du niveau $\mathrm{L}$ de la nouvelle aire ouverte durant cette campagne 2019. Cette première sélection nous a permis d'identifier, à un niveau macroscopique, les différents états de surface représentés, la diversité des matières premières et les caractéristiques de l'outillage dans ses grandes lignes.

15 L'observation directe au microscope numérique a nécessité d'effectuer une seconde sélection afin de réduire le nombre de pièces à une quantité analysable dans le temps de la fouille. Cet échantillonnage a répondu à deux critères :

- observer plusieurs individus de chaque matière première ;

- observer plusieurs individus de chaque grand type d'outil défini par l'analyse technofonctionnelle.

$16 \mathrm{Au}$ total, 29 artefacts ont été étudiés provenant majoritairement du niveau F-deb 1 $(n=23)$ et dans une moindre mesure du niveau F-Deb $2(n=2)$ et du niveau L-All $4(n=4)$. Concernant l'outillage, l'échantillon analysé a rassemblé des trièdres, des petits becs, des denticulés, des pièces présentant un bord retouché ou non potentiellement tranchant, et des petits éclats de retouche ou d'encoche.

17 Les états de surface des pièces montrent des altérations de degré d'intensité très variable. Dans la majorité des cas, on observe un lustre d'aspect assez brillant, lisse et homogène. Sur les pièces les plus altérées, ce lustre s'accompagne d'un émoussé des nervures et bords. De rares pièces, souvent en silex à grain fin, présentent aussi une patine blanche. Les artefacts en calcaire quant à eux comportent systématiquement une désilicification de la matière. Enfin, certains supports, moins fréquents, ne montrent aucune altération notable: les nervures et bords sont frais, coupants, sans patine ni lustre. 

sont variés. On note des esquillements, souvent isolés, parfois plus denses et continus. Fréquemment, un écrasement important du fil a été constaté (fig. $5, n^{\circ} 3$ ). En l'état, il est difficile d'exclure complètement une cause taphonomique pour l'expliquer mais leur présence, loin d'être anecdotique reste à analyser plus en détail. Les émoussés sont quant à eux plus rares. Par ailleurs, ils n'ont été pris en considération que lorsque la pièce ne présentait aucun émoussé de nervure ou de surface.

21 Sur les autres types d'outils, notamment sur les petits éclats de confection et les éclats de coche, des esquillements continus à superposés ont été notés sur certaines portions de bord. 
Fig. 5 - Traces observées à la Dino-lite (High Speed real time), grossissement de 10x à 220x.
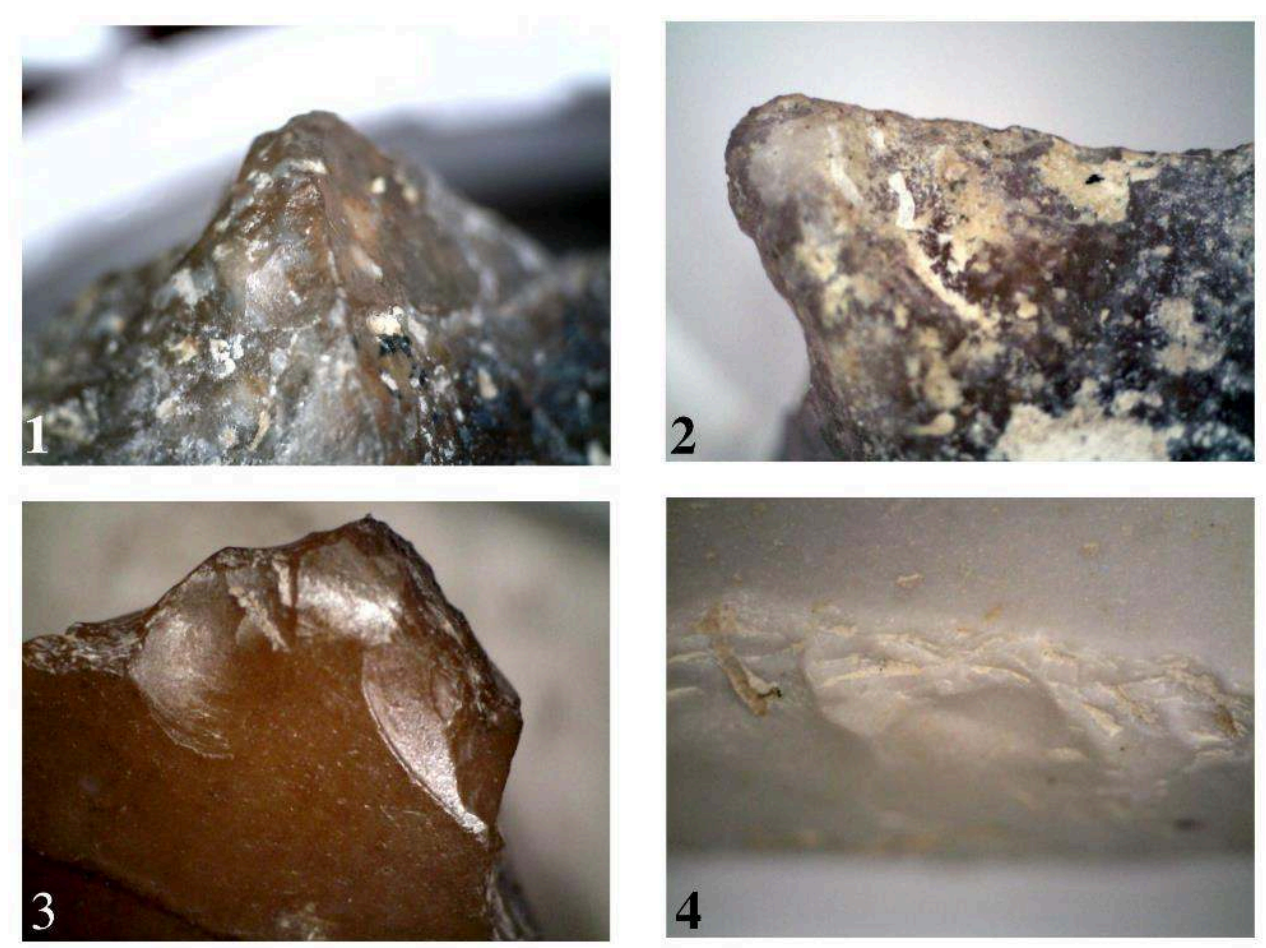

1 : Esquillements à terminaisons réfléchies dans le sens de la nervure, $\times 50$ (pièce $n^{\circ} 294-F-$ deb 1$): 2$ : Esquillements sur les bords latéraux du trièdre et écrasement du fil, $\times 50$ (pièce no 313 -F-deb 1) ; 3 : Esquillements sur les bords latéraux du trièdre et léger émoussé, $\times 40$ (pièce n 688-L-all4) ; 4 : Fort écrasement du fil sur le bord d'un outil retouché, x75 (pièce n³123-F-deb1).

Photo A. Da Costa/EFR Sabap Basilicata.

L'ensemble des traces observées nous conduit à conclure qu'une étude tracéologique est possible sur l'assemblage des différents niveaux d'Atella. Cette première observation du matériel est donc positive puisqu'elle met en évidence la présence de traces, notamment des micro-esquillements, des écrasements et quelques émoussés observables de façon récurrente sur certaines parties transformatives d'outils, tels que les trièdres. Toutefois, la forte présence d'altérations visibles sur les états de surface amène à observer chaque pièce dans le détail et à mettre en place pour la suite une grille d'observation et d'analyse spécifique à appliquer sur chaque artefact. La compréhension des dynamiques de déposition des niveaux à l'échelle du site permettra également de mieux appréhender la diversité des états de surface observés.

L'utilisation d'un microscope optique, avec des grossissements jusqu'à 400 ou 500x, s'avère également nécessaire pour mener à bien l'identification des traces d'utilisation, ajoutant ainsi des informations sur la présence de polis et/ou de stries et autorisant une meilleure caractérisation de la morphologie des micro-esquillements déjà notés.

\section{Le matériel archéologique}

Le matériel archéologique des niveaux historiques est principalement constitué de fragments de céramique, de tuiles et de rares éléments en silex et en métal. Selon une observation très préliminaire, certains de ces éléments, en particulier des fragments de céramique peinte en noir, pourraient être d'époque lucanienne. 
Dans les niveaux paléolithiques, peu de matériel a été découvert étant donnée la nature de la mission 2019. Si au total 411 pièces ont été répertoriées surtout dans les sondages 2 et 3, la plupart sont de très petites dimensions et sont issues du tamisage (tabl. 1). Étant donné le petit nombre de pièces, il est difficile de décrire avec précision les chaînes d'exploitation. Cependant, on peut observer de manière préliminaire que le matériel lithique présente les mêmes caractéristiques que ce qui est déjà connu à Atella, à savoir des éclats (fig. $\mathbf{6}, \mathrm{n}^{\text {os }} \mathbf{1}, \mathbf{2}$ ), des petits outils (fig. $6, \mathbf{n}^{\circ} \mathbf{3}$ ) et des petits éclats de confection et de retouche (fig. 6, $\mathbf{n}^{\circ} 4$ ). Concernant les aspects taphonomiques, l'état de surface des pièces issues des sondages 2 et 3 semble plus frais que celui de l'ensemble $\mathrm{F}$ (Deb 1 et 2 ). De plus, la présence importante de très petits éléments trouvés au tamisage semble indiquer que l'ensemble $\mathrm{L}$ offre un potentiel prometteur en ce qui concerne la concentration du matériel et l'état de conservation des niveaux archéologiques.

Fig. 6 - Cimitero di Atella, matériel lithique mission 2019.

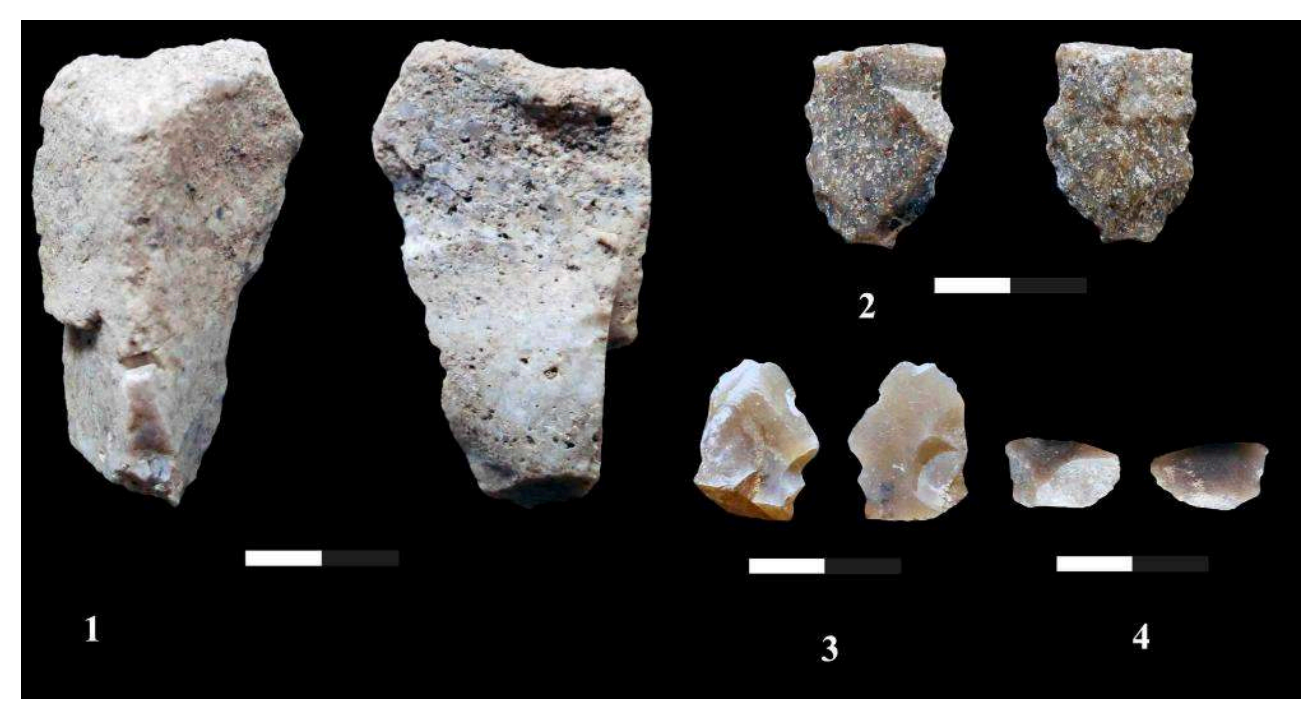

1, 2 : éclats en silex ; 3 : petit outil ; 4 : éclat d'encoche.

Photo L. Germond et L. Clos/EFR Sabap Basilicata.

\section{Perspectives}

Les premières années de fouille avaient permis de confirmer l'âge du gisement, de préciser la nature des enregistrements sédimentaires et de réévaluer les chaines opératoires de production lithiques et en 2017 et 2018 nous avions entamé la fouille d'un niveau archéologique inédit ${ }^{23}$. En effet, la fouille du sommet de l'ensemble $F$, précédant l'arrivée de cendres volcaniques a permis de mettre au jour une surface qui apparaît peu perturbée, riche en matériel lithique et faunique bien conservé.

En 2019, l'ouverture d'une nouvelle aire de fouille a permis d'évaluer l'extension du site et d'avoir un aperçu de la concentration et de l'état de conservation des occupations de l'ensemble L. Deux niveaux archéologiques ont été identifiés dans ces dépôts d'origine fluviatile, dont un relativement riche malgré la faible surface sondée, portant à 4 le nombre total des niveaux d'occupation conservés à Atella. Un premier examen microscopique sur un échantillon d'outils en pierre issus des niveaux les moins 
perturbés (au sommet et à la base d'un niveau de tephra) a permis de mettre en évidence la présence de traces d'utilisation.

La poursuite des travaux à Atella, qui dépendra de l'avancée du projet de muséalisation, devra donc se concentrer sur les trois principaux niveaux identifiés, à savoir le sommet du tephra I (base de l'ensemble L), la base du tephra I (sommet de l'ensemble F) et, quand les conditions le permettront, sur la base de l'ensemble F qui n'a pas encore été fouillée mais qui apparaît riche en matériel dans un petit secteur de l'aire Borzatti. La partie supérieure de la séquence, n'ayant pas livré de concentration significative de matériel ni dans l'aire Borzatti ni dans la nouvelle aire, pourra être rapidement fouillée par des moyens mécaniques.

Après plusieurs années de recherches à Atella, nous avons donc pour la première fois une vision globale du potentiel archéologique du site. La jonction entre la nouvelle et l'ancienne aire de fouille s'avère désormais nécessaire pour mettre en place une fouille extensive des niveaux d'occupations identifiés. Ainsi, la question des changements techniques pendant cette phase ancienne de l'Acheuléen pourra être abordée à travers trois occupations sub-contemporaines dans leurs dimensions techniques, économiques et spatiales.

Tableau 1 - Matériel archéologique de la mission 2019.

Image 10000000000003E00000019DA0BE4FEF62502562.jpg

\section{BIBLIOGRAPHIE}

Abruzzese - Aureli - Rocca $2016=$ C. Abruzzese, D. Aureli, R. Rocca, Assessment of the Acheulean in Southern Italy: New study on the Atella site (Basilicata, Italy), dans Quaternary International, 393, 2016, p. $158-168$.

Arzarello - Peretto $2010=$ M. Arzarello, C. Peretto, Out of Africa: The first evidence of Italian peninsula occupation, dans Quaternary International, 223-224, 2010, p. 65-70.

Arzarello - De Weyer - Peretto 2016 = M. Arzarello, L. De Weyer, C. Peretto, The first European peopling and the Italian case: Peculiarities and "opportunism", dans Quaternary International, 393, 2016, p. 41-50.

Aureli et al. 2016 = D. Aureli, R. Rocca, C. Lemorini, V. Modesti, S. Scaramucci, S. Milli, B. Giaccio, F. Marano, M.R. Palombo, A. Contardi, Mode 1 or mode 2? "Small tools" in the technical variability of the European Lower Palaeolithic: The site of Ficoncella (Tarquinia, Lazio, central Italy), dans Quaternary International, 393, 2016, p. 169-184.

Barsky - de Lumley 2010 = D. Barsky, H. de Lumley, Early European Mode 2 and the stone industry from the Caune de l'Arago's archeostratigraphical levels "P", dans Quaternary International, 223-224, 2010, p. 71-86.

Borzatti von Löwenstern 1998 = E. Borzatti von Löwenstern, Il Bacino di Atella nella Preistoria, dans Studi per l'ecologia del Quaternario, 20, 1998, p. 7-39. 
Borzatti von Löwenstern et al. 1990 = E. Borzatti von Löwenstern, M. Sozzi, S. Vannucci, F. Vianello, L'acheuleano del cimitero di Atella, dans Prime indagini sulla stratigrafia del sedimento $e$ sulle industrie litiche, dans Studi per l'ecologia del Quaternario, 12, 1990, p. 9-30.

Borzatti von Löwenstern et al. 1997 = E. Borzatti von Löwenstern, A. Palchetti, M. Sozzi, M. Maestrini, Témoignages de l'Acheuléen inférieur en Italie méridionale : Le gisement du Cimitero di Atella (Basilicata), dans L'Anthropologie, 101-4, 1997, p. 617-638.

Carbonell et al. 1999 = E. Carbonell, M. Mosquera, X.P. Rodríguez, R. Sala, J. van der Made, Out of Africa: The dispersal of the earliest technical systems reconsidered, dans Journal of Anthropological Archaeology, 18-2, 1999, p. 119-136.

Carbonell - Rodríguez 2006 = E. Carbonell, X.P. Rodríguez, The first human settlement of Mediterranean Europe, dans Comptes Rendus Palevol, 5-1/2, 2006, p. 291-298.

Ciolli $1997=$ N. Ciolli, Elephas antiquus Falconer \& Cautley del Cimitero di Atella (Pz), dans Studi per l'ecologia del Quaternario, 19, 1997, p. 25-34.

Di Leo et al. 2011 = P. Di Leo, D. Gioia, C. Martino, A. Pappalardo, M. Schiattarella, Geomorphological, mineralogical, and geochemical evidence of Pleistocene weathering conditions in the southern Italian Apennines, dans Geologica Carpathica, 62-1, 2011, p. 43-53.

Di Muro 1999 = A. Di Muro, Inquadramento tefrostratigrafico del sito acheuleano del Cimitero di Atella (Basilicata-Italia), dans Studi per l'ecologia del Quaternario, 21, 1999, p. 7-14.

Gallotti - Peretto 2015 = R. Gallotti, C. Peretto, The Lower/early Middle Pleistocene small débitage productions in Western Europe: New data from Isernia La Pineta t. 3c (Upper Volturno Basin, Italy), dans Quaternary International, 357, 2015, p. 264-281.

García-Medrano et al. 2014 = P. García-Medrano, A. Ollé, M. Mosquera, I. Cáceres, C. Díez, E. Carbonell, The earliest Acheulean technology at Atapuerca (Burgos, Spain): Oldest levels of the Galería site (GII Unit), dans Quaternary International, 353, 2014, p. 170-194.

Giannandrea et al. 2006 = P. Giannandrea, L. La Volpe, C. Principe, M. Schiattarella, Unità stratigrafiche a limiti inconformi e storia evolutiva del vulcano medio-pleistocenico di Monte Vulture (Appennino meridionale, Italia), dans Bollettino-Società Geologica Italiana, 125-1, 2006, p. 67-92.

Moncel et al. 2013 = M.-H. Moncel, J. Despriée, P. Voinchet, H. Tissoux, D. Moreno, J.-J. Bahain, G. Courcimault, C. Falguères, Early evidence of Acheulean settlement in Northwestern Europe - La Noira Site, a 700000 year-old occupation in the Center of France, dans PLOS ONE, 8, 11, 2013, p. e75529.

Moncel et al. 2018 = M.-H. Moncel, M. Arzarello, É. Boëda, S. Bonilauri, B. Chevrier, C. Gaillard, H. Forestier, L. Yinghua, F. Sémah, V. Zeitoun, Assemblages with bifacial tools in Eurasia (third part). Considerations on the bifacial phenomenon throughout Eurasia, dans Comptes Rendus Palevol, 17-1, 2018, p. 77-97.

Moncel et al. 2019 = M.-H. Moncel, C. Santagata, A. Pereira, S. Nomade, J.-J. Bahain, P. Voinchet, M. Piperno, A biface production older than $600 \mathrm{ka}$ ago at Notarchirico (Southern Italy) contribution to understanding early Acheulean cognition and skills in Europe, dans PLOS ONE, 14-9, 2019, p. e0218591.

Mosquera et al. 2016 = M. Mosquera, A. Ollé, P. Saladié, I. Cáceres, R. Huguet, A. Rosas, J. Villalaín, A. Carrancho, D. Bourlès, R. Braucher, A. Pineda, J. Vallverdú, The Early Acheulean technology of Barranc de la Boella (Catalonia, Spain), dans Quaternary International, 393, 2016, p. 95-111.

Mosquera et al. 2018 = M. Mosquera, A. Ollé, X.P. Rodríguez-Álvarez, E. Carbonell, Shedding light on the Early Pleistocene of TD6 (Gran Dolina, Atapuerca, Spain): The technological sequence and occupational inferences, dans PLOS ONE, 13-1, 2018, p. e0190889. 
Nicoud 2011 = E. Nicoud, Le phénomène acheuléen en Europe occidentale : Approche chronologique, technologie lithique et implications culturelles, thèse de doctorat, université Aix-Marseille 1, Aix-enProvence, 2011.

Nicoud et al. 2016 = E. Nicoud, D. Aureli, M. Pagli, V. Villa, C. Chaussé, S. Agostini, J.-J. Bahain, G. Boschian, J.-P. Degeai, F. Fusco, B. Giaccio, M. Hernandez, C. Kuzucuoglu, C. Lahaye, C. Lemorini, N. Limondin-Lozouet, P. Mazza, N. Mercier, S. Nomade, A. Pereira, V. Robert, M.A. Rossi, C. Virmoux, A. Zupancich, Preliminary data from Valle Giumentina Pleistocene site (Abruzzo, Central Italy): A new approach to a Clactonian and Acheulian sequence, dans Quaternary International, 409, Part B, 2016, p. 182-194.

Pereira 2017 = A. Pereira, Apport de la datation 40Ar/39Ar à la compréhension de l'évolution culturelle des pré-néanderthaliens en Italie centrale et méridionale entre 750 et $250 \mathrm{ka}$, thèse de doctorat, Muséum National d'Histoire naturelle de Paris, université de Ferrare, Paris-Ferrare, 2017.

Pereira et al. 2015= A. Pereira, S. Nomade, P. Voinchet, J.-J. Bahain, C. Falguères, H. Garon, D. Lefèvre, J.-P. Raynal, V. Scao, M. Piperno, The earliest securely dated hominin fossil in Italy and evidence of Acheulian occupation during glacial MIS 16 at Notarchirico (Venosa, Basilicata, Italy), dans Journal of Quaternary Science, 30-7, 2015, p. 639-650.

Pereira et al. 2017 = A. Pereira, S. Nomade, J.-J. Bahain, M. Piperno, Datation par 40Ar/39Ar sur monocristaux de feldspaths potassiques : exemple d'application sur le site pléistocène moyen ancien de Notarchirico (Basilicate, Italie), dans Quaternaire. Revue de l'Association française pour l'étude du Quaternaire, 28-2, 2017, p. 149-154.

Peretto et al. 2015 = C. Peretto, J. Arnaud, J. Moggi-Cecchi, G. Manzi, S. Nomade, A. Pereira, C. Falguères, J.-J. Bahain, D. Grimaud-Hervé, C. Berto, B. Sala, G. Lembo, B. Muttillo, R. Gallotti, U.T. Hohenstein, C. Vaccaro, M. Coltorti, M. Arzarello, A human deciduous tooth and new $40 \mathrm{Ar} / 39 \mathrm{Ar}$ dating results from the Middle Pleistocene archaeological site of Isernia La Pineta, Southern Italy, dans PLOS ONE, 10-10, 2015, p. e0140091.

Piperno 1999 = M. Piperno (dir.), Notarchirico: un sito del Pleistocene medio antico nel bacino di Venosa, Venosa, 1999.

Rocca 2016 = R. Rocca, Depuis l'Est ? Nouvelles perspectives sur les premières dynamiques de peuplement en Europe, dans L'Anthropologie, 120-3, 2016, p. 209-236.

Rocca - Abruzzese - Aureli 2016 = R. Rocca, C. Abruzzese, D. Aureli, European Acheuleans: Critical perspectives from the East, dans Quaternary International, 411, Part B, 2016, p. 402-411.

Rocca et al. 2016 = R. Rocca, C. Abruzzese, D. Aureli, J.-J. Bahain, P. Beneduce, P. Di Leo, P. Giannandrea, S.I. Giano, D. Gioia, D. Magri, S. Nomade, A. Pereira, M. Schiattarella, R. Sulpizio, P. Voinchet, New multidisciplinary research project on the Lower Palaeolithic site of Cimitero di Atella (Potenza), dans F. Negrino, F. Fontana, A. Moroni, J. Riel-Salvatore (dir.), The Palaeolithic and Mesolithic in Italy: new research and perspectives DAFIST, Genoa (Italy) - 4th-5th February 2016, Florence, 2016, p. 21-22.

Rocca - Aureli - Abruzzese $2018=$ R. Rocca, D. Aureli, C. Abruzzese, Cimitero di Atella. Nouvelles recherches et valorisation d'un gisement du Paléolithique ancien en Méditerranée, dans Chronique des activités archéologiques de l'École française de Rome, 2018.

Rocca - Aureli 2019 = R. Rocca, D. Aureli, Cimitero di Atella. Résultats de la mission 2018, dans Chronique des activités archéologiques de l'École française de Rome, 2019. 
Santagata 2016 = C. Santagata, Operating systems in units B and E of the Notarchirico (Basilicata, Italy) ancient Acheulean open-air site and the role of raw materials, dans Quaternary International, 411, Part B, 2016, p. 284-300.

Schiattarella et al. 2005 = M. Schiattarella, P. Beneduce, P. Di Leo, S.I. Giano, P. Giannandrea, C. Principe, Assetto strutturale ed evoluzione morfotettonica quaternaria del vulcano del Monte Vulture (Appennino Lucano), dans Bollettino-Società Geologica Italiana, 124-3, 2005, p. 543.

Villa - Buettner 2009 = I.M. Villa, A. Buettner, Chronostratigraphy of Monte Vulture volcano (southern Italy): secondary mineral microtextures and 39Ar-40Ar systematics, dans Bull Volcanol, 71, 10, 2009, p. $1195-1208$

Zucchelli $1999=$ M. Zucchelli, Bos primigenius Bojanus del Bacino di Atella (Potenza), dans Studi per l'ecologia del Quaternario, 21, 1999, p. 19-30.

Zucchelli $2000=$ M. Zucchelli, Nuovi ritrovamenti di resti osteologici di Bos primigenius Bojanus nel Bacino di Atella (Potenza), dans Studi per l'ecologia del Quaternario, 22, 2000, p. 11-22.

Zucchelli $2002=$ M. Zucchelli, I cervidi dell'Acheuleano antico del Cimitero di Atella (Potenza), dans Studi per l'ecologia del Quaternario, 24, 2002, p. 3-17.

\section{NOTES}

1. Borzatti von Löwenstern et al. 1997.

2. Pereira 2017 ; Rocca et al. 2016.

3. Abruzzese - Aureli - Rocca 2016 ; Rocca et al. 2016.

4. Rocca - Aureli - Abruzzese 2018 ; Rocca - Aureli 2019.

5. Borzatti von Löwenstern et al. 1997 ; Abruzzese - Aureli - Rocca 2016; Rocca - Aureli Abruzzese 2018.

6. Carbonell et al. 1999 ; Carbonell - Rodríguez 2006 ; Arzarello - De Weyer - Peretto 2016; Arzarello - Peretto 2010.

7. Moncel et al. 2013, 2018 et 2019 ; García-Medrano et al. 2014 ; Mosquera et al. 2016 et 2018.

8. Moncel et al. 2019 ; Nicoud 2011 ; Piperno 1999 ; Santagata 2016.

9. Pereira 2017 ; Pereira et al. 2015 et 2017.

10. Aureli et al. 2016 ; Galotti - Peretto 2015 ; Nicoud et al. 2016 ; Peretto et al. 2015.

11. Rocca - Aureli 2018.

12. Borzatti von Löwenstern 1998 ; Borzatti von Löwenstern et al. 1990 et 1997.

13. Ciolli 1997 ; Zucchelli 1999, 2000 et 2002.

14. Borzatti von Löwenstern et al. 1990 et 1997.

15. Rocca - Aureli - Abruzzese 2018.

16. Pereira 2017.

17. Di Muro 1999 ; Schiattarella et al. 2005 ; Giannandrea et al. 2006 ; Villa - Buettner 2009 ; Di Leo et al. 2011 ; Rocca et al. 2016 ; Rocca - Aureli - Abruzzese 2018.

18. Rocca - Aureli - Abruzzese 2018.

19. Abruzzese - Aureli - Rocca 2016 ; Rocca - Abruzzese - Aureli 2018.

20. Aureli et al. 2016 ; Rocca - Abruzzese - Aureli 2016 ; Rocca 2016.

21. Barsky - de Lumley 2010 ; Gallotti - Peretto 2015 ; Mosquera et al. 2018 ; Rocca - Abruzzese Aureli 2018 ; Santagata 2016.

22. Abruzzese - Aureli - Rocca 2016 ; Rocca - Abruzzese - Aureli 2017 ; Rocca et al. en cours.

23. Rocca - Abruzzese - Aureli 2018 ; Rocca - Aureli 2019. 


\section{RÉSUMÉS}

Le site paléolithique ancien d'Atella (Basilicate, Italie) a été découvert puis fouillé par E. Borzatti pendant une vingtaine d'années. Les travaux sur ce site ont repris au milieu des années 2000 dans le cadre d'un nouveau projet qui vise à comprendre les mécanismes des premiers peuplements de l'Europe méditerranéenne. Les premières années du projet ont été consacrées à la révision du contexte chrono-stratigraphique et à l'attribution techno-culturelle des occupations de Cimitero di Atella. En 2019, la mission a été principalement consacrée à l'ouverture d'une nouvelle aire de fouille, permettant d'évaluer l'extension du gisement.

The Lower Paleolithic site of Atella in Basilicata (Basilicata, Italy) was discovered and excavated by E. Borzatti for around twenty years. Work on this site resumed in the mid-2000s as part of a new project which aims to understand the mechanisms of the first settlements in Mediterranean Europe. The first years of the project were devoted to revising the chrono-stratigraphic context and the techno-cultural attribution of the occupations of Cimitero di Atella. In 2019, the mission was mainly devoted to the opening of a new excavation area, to assess the extension of the deposit

\section{INDEX}

institutions École française de Rome, Université Paris 1 Panthéon-Sorbonne, Université de la Basilicate, Umr 7041-ArScAn

Index géographique : Atella

Mots-clés : Paléolithique ancien, industrie lithique, Acheuléen, Italie, Basilicate

Keywords : Lower Palaeolithic, lithic industry, Acheulean, Italy, Basilicata

\section{AUTEURS}

\section{ROXANE ROCCA}

Université Paris 1 Panthéon-Sorbonne et UMR 7041-ArScan, EP - roxane.rocca@univ-paris1.fr

\section{AMÉLIE DA COSTA}

UMR 7041-ArScan, AnTET

\section{LUCIE GERMOND}

Université Paris-Nanterre et UMR 7041-ArScan, AnTET

\section{DANIELE AURELI}

Università di Siena et UMR 7041-ArScan, AnTET - danieleaureli1@gmail.com 